\title{
GLOBALIZATIONS OF HARISH-CHANDRA MODULES
}

\author{
WILFRIED SCHMID AND JOSEPH A. WOLF
}

1. Introduction. Let $G$ be a reductive Lie group, $\mathfrak{g}$ the complexification of its real Lie algebra $\mathfrak{g}_{0}$, and $K$ a maximal compactly embedded subgroup of $G$. In order to study various classes of representations $\left(\pi, V_{\pi}\right)$ of $G$ one makes use of the associated Harish-Chandra module $\left(\pi_{K}, V\right)$. On the other hand, a Harish-Chandra module $V$ can globalize to a representation of $G$ in a variety of ways. Among these is the maximal globalization [7], which depends on $V$ in a canonical manner. Here we show that several geometric constructions of representations of $G$ are equivalent, all leading to the maximal globalization of certain standard derived functor modules.

For simplicity of exposition we describe our result only for groups $G$ of Harish-Chandra class. It also holds for the larger class [9] of reductive groups.

2. The cohomologies. Consider the datum $(H, \chi, \mathfrak{b})$ where $H$ is a $\theta$ stable Cartan subgroup of $G, \mathfrak{b}$ is a Borel subalgebra of $\mathfrak{g}$ that contains $\mathfrak{h}$, and $\chi$ is a finite-dimensional irreducible representation of $H$. View the representation space $E_{\chi}$ as a $(\mathfrak{b}, H)$-module and let $\mathbf{E}_{\chi}$ denote the associated homogeneous vector bundle over $G / H$. Let $\mathfrak{n}=[\mathfrak{b}, \mathfrak{b}]$, the nilradical of $\mathfrak{b}$. One can attach a complex of $\mathbf{E}_{\chi}$-valued differential forms to the datum $(H, \chi, \mathfrak{b})$, for which the global sections are given by

$$
\left(C^{\infty}(G) \otimes E_{\chi} \otimes \Lambda \mathfrak{n}^{*}\right)^{H}, d .
$$

Here $H$ and $\mathfrak{n}$ act on $C^{\infty}(G)$ by right translation, the superscript $H$ refers to the space of $H$-invariants, and $d$ is the coboundary operator for Lie algebra cohomology of $\mathfrak{n}$.

REMARK 1. The sheaf version of the complex (2.1) is not, in general, acyclic. Thus (2.1) computes the hypercohomology of a complex of sheaves on $G / H$ rather than the cohomology of a single sheaf.

By taking $K$-finite $\mathbf{E}_{\chi}$-valued differential forms whose coefficients are formal power series, one obtains a formal analog of (2.1). The cohomology groups of this formal complex are the standard Zuckerman modules $I^{p}\left(G / H, \mathbf{E}_{\chi}\right)$. See $[\mathbf{3}]$.

Let $\mathbf{A}\left(G / H, \mathbf{E}_{\chi}\right)$ denote the complex that is the analog of (2.1) with smooth functions replaced by hyperfunctions,

$$
\mathbf{A}\left(G / H, \mathbf{E}_{\chi}\right): \quad\left(B(G) \otimes E_{\chi} \otimes \Lambda \mathfrak{n}^{*}\right)^{H}, d .
$$

The flag variety $X$ (of Borel subalgebras of $\mathfrak{g}$ ) contains $S=G \cdot \mathfrak{b}$ as a real analytic submanifold. Since $H$ normalizes $\mathfrak{b}, G / H$ maps $G$-equivariantly onto

Received by the editors November 1, 1986.

1980 Mathematics Subject Classification (1985 Revision). Primary 22E46, 22E47; Secondary 32M10.

First author supported by NSF Grant DMS-8317436; second author supported by NSF Grant DMS-8513467. 
$S$. The vector bundle $\mathbf{E}_{\chi}$ drops to a bundle on $S$, which we denote by the same symbol. There is a unique g-equivariant holomorphic extension $\tilde{\mathbf{E}}_{\chi} \rightarrow \tilde{S}$ to the germ of a neighborhood of $S$ in $X$. By homogeneity, $S$ has constant $C R$ dimension, so we can look at the induced $\bar{\partial}$ complex on $S$ as a submanifold of $X$, with hyperfunction coefficients

$$
\mathbf{B}\left(S, \mathbf{E}_{\chi}\right): \quad \tilde{\mathbf{E}}_{\chi} \text {-valued }(0, *) \text {-forms on } S \text { with coefficients in } B(S) .
$$

Finally, we consider the relative complex

Dolbeault complex of $\tilde{\mathbf{E}}_{\chi} \rightarrow \tilde{S}$, except that the

coefficients are hyperfunctions on $\tilde{S}$ with support on $S$.

Then $H^{p}\left(\mathbf{C}\left(S, \mathbf{E}_{\chi}\right)\right)$ is the local cohomology $H_{S}^{p}\left(X, O\left(\tilde{\mathbf{E}}_{\chi}\right)\right)$ of the sheaf $O\left(\tilde{\mathbf{E}}_{\chi}\right)$ along $S$.

\section{Statement of result.}

THEOREM. There are natural topological isomorphisms between

(1) $H^{q}\left(\mathbf{A}\left(G / H, \mathbf{E}_{\chi}\right)\right)$,

(2) $H^{q}\left(\mathbf{B}\left(S, \mathbf{E}_{\chi}\right)\right)$

(3) $H^{q+r}\left(\mathbf{C}\left(S, \mathbf{E}_{\chi}\right)\right)=H_{S}^{q+r}\left(X, O\left(\tilde{\mathbf{E}}_{\chi}\right)\right)$ where $r=\operatorname{dim}_{C} X-\operatorname{dim}_{C R} S$, and

(4) the maximal globalization $I^{q}\left(G / H, \mathbf{E}_{\chi}\right)_{\max }$ of the standard Zuckerman module $I^{q}\left(G / H, \mathbf{E}_{\chi}\right)$.

REMARK 2. The complexes (2.2), (2.3), and (2.4) do not have good intrinsic topologies because there is no reasonable topology on the space of hyperfunctions on a noncompact manifold. The topological part of the statement of the theorem must be interpreted in one of two (equivalent) ways. First, the proof provides the appropriate topology. Second, topology is determined by the underlying Harish-Chandra module $I^{q}\left(G / H, \mathbf{E}_{\chi}\right)$, since the topology of the maximal globalization can be defined purely in algebraic terms. See [7].

REMARK 3. In the case of a compact Cartan subgroup $H$, the theorem is due to R. Aguilar-Rodriguez [1]; see $\S 4 \mathrm{~A}$ below. We are informed that there may be some overlap between our work and work in progress of $\mathrm{H}$. Hecht and J. Taylor on geometric realizations of the minimal globalization of a Harish-Chandra module.

\section{Indication of proof.}

4A. COMPACT CARTAN Subgroup. Let $\mathfrak{h} \subset \mathfrak{k}$. Then $\mathfrak{b} \cap \overline{\mathfrak{b}}=\mathfrak{h}$, so $S$ is open in $X$ and $r=0$, and $S=G / H$ as homogeneous space. Each of $H^{q}\left(\mathbf{A}\left(G / H, \mathbf{E}_{\chi}\right)\right), H^{q}\left(\mathbf{B}\left(S, \mathbf{E}_{\chi}\right)\right)$, and $H_{S}^{q+r}\left(X, O\left(\tilde{\mathbf{E}}_{\chi}\right)\right)$ is just the Dolbeault cohomology $H^{q}\left(S, O\left(\mathbf{E}_{\chi}\right)\right)$, which is the maximal globalization of its underlying Harish-Chandra module [7].

Suppose that $G$ is connected and let $\lambda$ be the differential of $\chi$. If $\lambda+\rho$ is nonsingular and antidominant, the Dolbeault cohomology has the same underlying Harish-Chandra module as a certain discrete series module [5], so its underlying Harish-Chandra module is the standard Zuckerman module $I^{q}\left(G / H, \mathbf{E}_{\chi}\right)[\mathbf{8}]$. One passes to general $\lambda$ by tensoring $[\mathbf{1}, \mathbf{1 0}]$. We extend the result to disconnected groups by the method of $[9]$. 
4B. ISOMORPHISM OF THE COHOMOLOGIES. Here $H$ is an arbitrary $\theta$ stable Cartan subgroup of $G$. Let $P=M A N_{0}$ denote the associated cuspidal parabolic subgroup of $G: H=T \times A$ and $Z_{G}(A)=M \times A$ under $\theta$. Then $N_{0}$ is the fibre of $G / H \rightarrow S$ and $M / T$ is the fibre of $p: S \rightarrow G / P$.

Compare the spectral sequences of $p: S \rightarrow G / P$ for the complexes (2.2) and (2.3). In each case, at the $E_{2}$ level one has Dolbeault cohomology $H^{*}\left(M / T, \mathcal{O}\left(\left.\mathbf{E}_{\chi}\right|_{M / T}\right)\right)$ along the fibre and de Rham cohomology, hyperfunction coefficients, along the base. We conclude that $H^{q}\left(\mathbf{A}\left(G / H, \mathbf{E}_{\chi}\right)\right) \cong$ $H^{q}\left(\mathbf{B}\left(S, \mathbf{E}_{\chi}\right)\right)$.

Locally, $S=\mathbf{C}^{k} \times \mathbf{R}^{r}$ in $X=\mathbf{C}^{k} \times \mathbf{C}^{r}$, the latter holomorphic in $\mathbf{C}^{r}$ but not necessarily in $\mathbf{C}^{k}, k=\operatorname{dim}_{C R} S$, with $p: S \rightarrow G / P$ given by $(z, t) \rightarrow t$. $B\left(\mathbf{R}^{r}\right)$ is just the local cohomology $H_{R}^{r}\left(\mathbf{C}^{r}, 0\right)$. So locally, thus globally, $\mathbf{B}\left(S, \mathbf{E}_{\chi}\right) \cong \mathbf{C}\left(S, \mathbf{E}_{\chi}\right)$ with dimension shifted by $r$. Conclusion:

$$
H^{q}\left(\mathbf{B}\left(S, \mathbf{E}_{\chi}\right)\right) \cong H^{q+r}\left(\mathbf{C}\left(S, \mathbf{E}_{\chi}\right)\right)=H_{S}^{q+r}\left(X, O\left(\tilde{\mathbf{E}}_{\chi}\right)\right) .
$$

The cohomologies (1), (2), and (3) of the theorem now are isomorphic.

4C. MAXIMALly REAL POlaRization. Here $H$ is an arbitrary $\theta$-stable Cartan subgroup of $G$ and the positive root system $\Phi^{+}$is chosen to maximize $\left|\Phi^{+} \cap \bar{\Phi}^{+}\right|=\operatorname{dim}_{R} \mathfrak{n}_{0}, \mathfrak{n}_{0}=\mathfrak{g}_{0} \cap \mathfrak{n}$. Again use the spectral sequence of $p: S \rightarrow$ $G / P$ for the complex (2.2) and the result of $\S 4 \mathrm{~A}$ to see that $I^{q}\left(G / H, \mathbf{E}_{\chi}\right)$ is the underlying Harish-Chandra module. On the other hand, the spectral sequence of $p: S \rightarrow G / P$ for (2.3) shows that we are looking at hyperfunction differential forms with values in a Fréchet bundle; that is the maximal globalization of the induced module [7]. In view of the result of $\S 4 \mathrm{~B}$ this proves the theorem in the case of a maximally real polarization.

4D. ANy POlarization, SOME Weyl Chamber. Given $H$, we pass from any positive root system to a maximally real positive root system by a sequence of operations $\Phi^{+} \rightarrow w_{\alpha}\left(\Phi^{+}\right)$where $\alpha$ is a simple complex root for $\Phi^{+}$and $w_{\alpha}$ is the Weyl reflection.

Suppose that we are in the situation just described. Then both $\bar{\alpha}$ and $w_{\alpha}(\bar{\alpha})$ are negative, and we consider the fibration $t: X \rightarrow X_{\alpha}$ where $X_{\alpha}$ is the flag of parabolic subalgebras of $\mathfrak{g}$ that are conjugate to $\mathfrak{b}_{\alpha}=\mathfrak{b}+\mathfrak{g}_{\alpha}$. Denote $S_{\alpha}=G \cdot \mathfrak{b}_{\alpha} \subset X_{\alpha}$, so $t^{-1}\left(S_{\alpha}\right)=S \cup S_{0}$ where $S_{0}=G \cdot w_{\alpha}(\mathfrak{b})$. Then $t$ is bijective from $S_{0}$ to $S_{\alpha}$ and the fibre $F=t^{-1}\left(\mathfrak{b}_{\alpha}\right) \cong P^{1}(\mathbf{C})$ with $F \cap S \cong \mathbf{C}$. Let $s$ and $z$ be local coordinates on $S$ and $F$, so $(s, z)$ is a local coordinate on $t^{-1}\left(S_{\alpha}\right)$ with $S_{0}$ given by $z=\varphi(s), \bar{\partial} \varphi \neq 0$ everywhere. If $H^{1}\left(F, O\left(\left.\mathbf{E}_{\chi}\right|_{F}\right)\right)=0$ then the Leray sequence of $\left.t\right|_{t^{-1}\left(S_{\alpha}\right)}$ gives

$$
H^{q}\left(\mathbf{B}\left(S, \mathbf{E}_{\chi}\right)\right) \cong H^{q-1}\left(\mathbf{B}\left(S_{\alpha}, R^{0} \mathbf{E}_{\chi}\right)\right) \cong H^{q-1}\left(\mathbf{B}\left(S_{0}, \mathbf{E}_{\chi+\alpha}\right)\right) \text {. }
$$

$\left(\rho\left(w_{\alpha} \Phi^{+}\right)=\rho\left(\Phi^{+}\right)-\alpha\right.$ and $\chi$ shifts to compensate. $)$ What we have just done is the counterpart of the Beilinson-Bernstein intertwining functor $[2$, 4]. An analogous argument [3] gives the same isomorphism for the standard Zuckerman modules.

Now suppose that the positive root system $\Phi^{+}$is given, and that $\left\{\alpha_{1}, \ldots\right.$, $\left.\alpha_{m}\right\}$ is a sequence of complex roots as above such that the product of the Weyl reflections carries $\Phi^{+}$to a maximally real positive root system. To carry out the isomorphisms just described, we need only ensure that $\lambda$ is not negative 
integral with respect to any of the $\alpha_{i}$. We may assume that all the $\alpha_{i}$ belong to $\Phi^{+}$, so that holds for all $\chi$ with differential $\lambda$ in some particular Weyl chamber.

Now, in view of $\S \S 4 \mathrm{~B}$ and $4 \mathrm{C}$, there is a Weyl chamber $\mathrm{D}$ such that the theorem holds whenever the differential of $\chi$ belongs to $\mathbf{D}$.

4E. ANY POlARIZATION, ANY WEYL CHAMBER. This is a tensoring argument. The point is that standard Zuckerman modules, and the maximal globalization functor, both behave well with respect to tensoring $[\mathbf{7}, \mathbf{8}, \mathbf{1 0}]$, so we need only show that the local cohomology $H_{S}^{q+r}\left(X, O\left(\tilde{\mathbf{E}}_{\chi}\right)\right)$ also behaves well. Arguing as in [6], that comes down to checking that the center $\mathbf{Z}(\mathfrak{g})$ of the universal enveloping algebra of $\mathfrak{g}$ acts on $H_{S}^{q+r}\left(X, \mathcal{O}\left(\tilde{\mathbf{E}}_{\chi}\right)\right)$ by the character that Harish-Chandra denotes $\chi_{\lambda+\rho}$. Local cohomology can be calculated from a relative covering using cocycles with coefficients that are holomorphic sections of $\tilde{\mathbf{E}}_{\chi}$, so this comes down to showing that $\mathbf{Z}(\mathfrak{g})$ acts on $O\left(\tilde{\mathbf{E}}_{\chi}\right)$ by $\chi_{\lambda+\rho}$. But a germ in $O\left(\tilde{\mathbf{E}}_{\chi}\right)$ is represented by a germ in $\left(C^{\infty}\left(G_{C}\right) \otimes E_{\chi} \otimes \Lambda \mathfrak{n}^{*}\right)^{H, \mathfrak{n}}$, and the Harish-Chandra homomorphism $\mathbf{Z}(\mathfrak{g}) \rightarrow \mathbf{U}(\mathfrak{h})$ gives the result.

This completes the indication of proof of the theorem.

\section{REFERENCES}

1. R. Aguilar-Rodriguez, Connections between representations of Lie groups and sheaf cohomology, Thesis, Harvard Univ., 1987.

2. A. Beilinson and J. Bernstein, A generalization of Casselman's submodule theorem, Representation Theory of Reductive Groups, Progress in Math., vol. 40, Birkhäuser, Boston, 1983, pp. 35-52.

3. H. Hecht, D. Milicić, W. Schmid and J. A. Wolf, Localization and standard modules for real semisimple Lie groups, I: The Duality Theorem (to appear).

4. __ Localization and standard modules for real semisimple Lie groups. II: Applications (to appear).

5. W. Schmid, Homogeneous complex manifolds and representations of semisimple Lie groups, Ph.D. thesis, Berkeley, 1967.

6. $L^{2}$ cohomology and the discrete series, Ann. of Math. (2) 103 (1976), 375-394.

7. __ Boundary value problems for group invariant differential equations, Proc. Cartan Symposium (Lyon, 1984), Asterix 1985, pp. 311-322.

8. D. Vogan, Representations of real reductive Lie groups, Progress in Math., vol. 15, Birkhäuser, Boston, 1981.

9. J. A. Wolf, Unitary representations on partially holomorphic cohomology spaces, Mem. Amer. Math. Soc., vol. 138, Amer. Math. Soc., Providence, R.I., 1974.

10. G. Zuckerman, Tensor products of finite and infinite dimensional representations of semisimple Lie groups, Ann. of Math. (2) 106 (1977), 295-308.

Department of Mathematics, Harvard University, Cambridge, MasSACHUSETTS 02138

Department of MAThematics, University of CALifornia at Berkeley, BERKELEY, CALIFORNIA 94720 